Provided for non-commercial research and education use. Not for reproduction, distribution or commercial use.

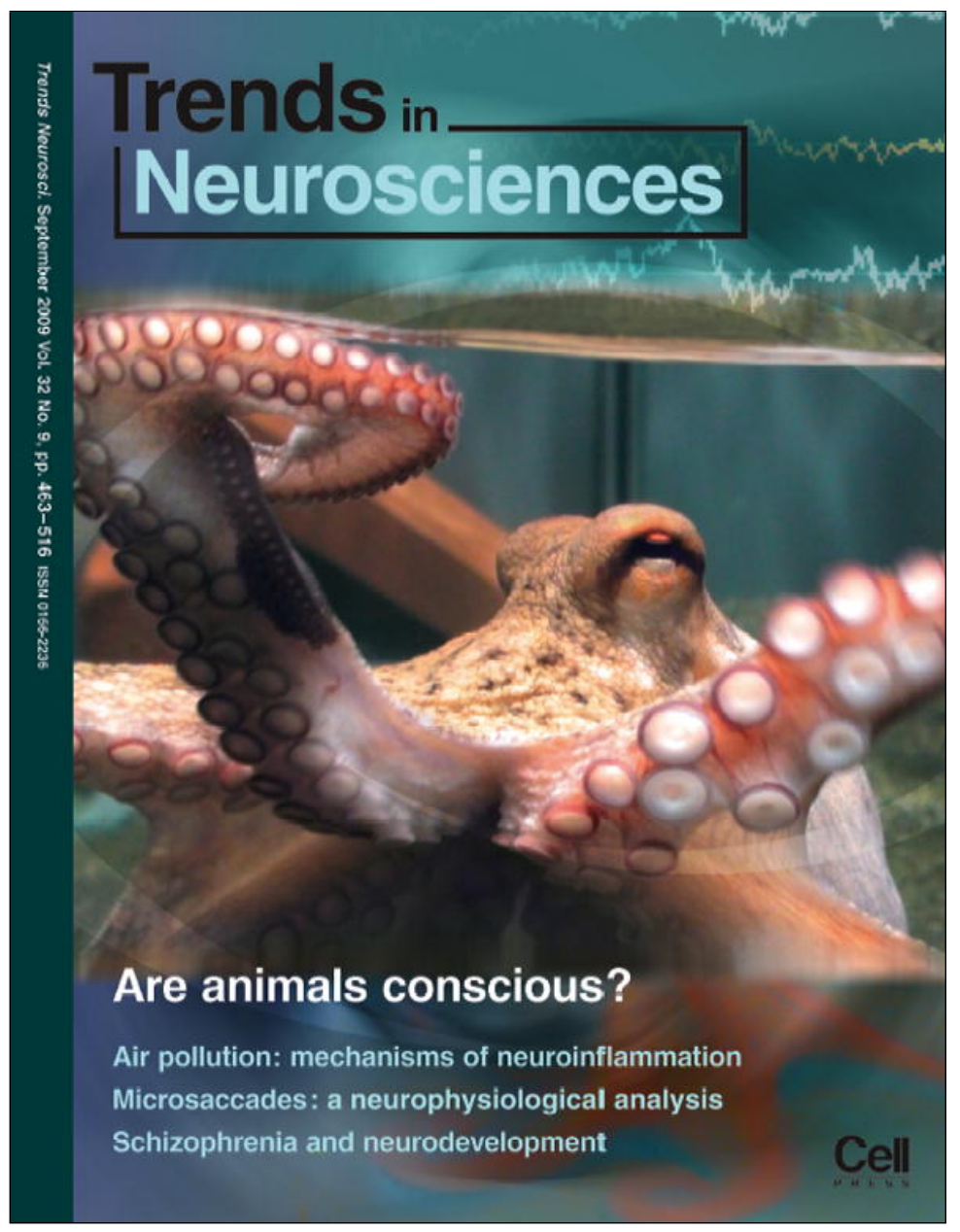

This article appeared in a journal published by Elsevier. The attached copy is furnished to the author for internal non-commercial research and education use, including for instruction at the authors institution and sharing with colleagues.

Other uses, including reproduction and distribution, or selling or licensing copies, or posting to personal, institutional or third party websites are prohibited.

In most cases authors are permitted to post their version of the article (e.g. in Word or Tex form) to their personal website or institutional repository. Authors requiring further information regarding Elsevier's archiving and manuscript policies are encouraged to visit:

http://www.elsevier.com/copyright 


\title{
Microsaccades: a neurophysiological analysis
}

\author{
Susana Martinez-Conde ${ }^{1}$, Stephen L. Macknik ${ }^{1}$, Xoana G. Troncoso ${ }^{1,2}$ and \\ David H. Hubel ${ }^{3}$
}

${ }^{1}$ Barrow Neurological Institute, Phoenix, AZ, USA

${ }^{2}$ Division of Biology, California Institute of Technology, Pasadena, CA, USA

${ }^{3}$ Department of Neurobiology, Harvard Medical School, Boston, MA, USA

\begin{abstract}
Microsaccades are the largest and fastest of the fixational eye movements, which are involuntary eye movements produced during attempted visual fixation. In recent years, the interaction between microsaccades, perception and cognition has become one of the most rapidly growing areas of study in visual neuroscience. The neurophysiological consequences of microsaccades have been the focus of less attention, however, as have the oculomotor mechanisms that generate and control microsaccades. Here we review the latest neurophysiological findings concerning microsaccades and discuss their relationships to perception and cognition. We also point out the current gaps in our understanding of the neurobiology of microsaccades and identify the most promising lines of enquiry.
\end{abstract}

\section{Introduction}

Eye movements are critical in piecing together a coherent visual perception of the world around us. Fixational eye movements, the microscopic and unnoticed motions of the eye made when fixing the gaze between larger eye movements, are perhaps the least understood of all eye movement types, despite their critical importance to normal vision. Classical studies have demonstrated that, in the absence of fixational eye movements, neural adaptation ensues and observers become functionally blind to stationary objects during fixation [1-4]. Fixational eye movements comprise microsaccades, drift and tremor. Table 1 lists microsaccade parameters in recent publications (Ref. [4] lists the characteristics of microsaccades, drift and tremor according to studies published in 2004 and earlier).

Microsaccades are the largest and fastest of the fixational eye movements. They contribute to maintaining visibility during fixation by shifting the retinal image in a fashion that overcomes adaptation, thus generating neural responses to stationary stimuli in visual neurons [4-6]. Recent discoveries have shown that microsaccades are critically related to many aspects of visual perception [6-11], attention and cognition [12-16] and are thus potentially very important in neurological and ophthalmic disease [17]. The last decade of research has seen a very rapid increase in the number of papers dedicated to microsaccades, with the highest increase in the last few years [18]. Once considered a mere nervous tic [19], microsaccades are today a central and fastgrowing topic of interest in the visual, oculomotor and cognitive neurosciences [20]. The field of inquiry spans the perceptual effects of microsaccades, the neural responses they evoke and their oculomotor generation mechanisms.

Our objectives in this review are three-fold: (1) to discuss the most recent advances in microsaccade research within a neurophysiological framework; (2) to analyze how discoveries in specific areas of microsaccade research (oculomotor, visual and cognitive neuroscience) impact on other findings and help in interpretation; and (3) to identify current trends in the field, their expected contributions in the near future and the most important gaps to address. We first focus on recent progress in microsaccade characterization, a technically complex task because of the microscopic nature of microsaccades and their overlap in physical characteristics with those of large or exploratory saccades. We then address the neural responses evoked by

\section{Glossary}

Bursts: Clusters of action potentials.

Covert attention: Attention directed to stimuli that are not at the center of gaze. Discrete sampling: Time-limited measurement of continuous time-varying data.

Drifts: Slow curvy motions that occur between microsaccades.

Endogenous attention: Attention that is directed voluntarily by top-down mechanisms.

Exogenous attention: Attention that is automatically drawn to a stimulus in a reflexive or bottom-up manner.

Inhibition of return: the phenomenon by which a stimulus presented at a recently attended location evokes a weaker reaction than a stimulus appearing at a location not yet attended.

Fixational eye movements: Involuntary eye movements produced during attempted fixation. Fixational eye movements in primates comprise microsaccades, drifts and tremor.

Microsaccades: Involuntary saccades produced during attempted fixation. They carry the retinal image across a range of several dozen to several hundred photoreceptor widths.

Spatial summation: The combination of two or more inputs arriving simultaneously through different synapses within a neuron's dendritic tree. Temporal summation: The combination of two or more inputs arriving nonsimultaneously through the same or different synapses within a neuron's dendritic tree.

Tremor: Very fast $(\sim 90 \mathrm{~Hz})$, extremely small oscillation (about the diameter of a foveal cone) superimposed on drifts.

Visual masking: A visible target (a visual stimulus, such as a rectangle) is rendered invisible by changing the context in which the target is presented without actually modifying the physical properties of the target itself. That is, the target becomes less visible due solely to its spatial and/or temporal context. 
Table 1. Physical parameters of microsaccades (as reported by original research studies in 2004-2009)

\begin{tabular}{|c|c|c|c|c|c|c|c|c|}
\hline Amplitude & Frequency & $\begin{array}{l}\text { Intersaccadic } \\
\text { Interval }\end{array}$ & Duration & Velocity & $\begin{array}{l}\text { Binocularity } \\
\text { required }\end{array}$ & Species & Eye Tracking & Reference \\
\hline $\max : 1.4^{\circ}$ & mean: $>2 \mathrm{~Hz}$ & & & $\begin{array}{l}\text { mean: } \\
4.43 \% \mathrm{~s}\end{array}$ & No & monkey & $\begin{array}{l}1000 \mathrm{~Hz} \text { Scleral } \\
\text { Search Coil }\end{array}$ & {$[50]$} \\
\hline $\max : 1^{\circ}$ & & & $\min : 16.8 \mathrm{~ms}$ & $\max : 200 \% \mathrm{~s}$ & No & human & $\begin{array}{l}238 \mathrm{~Hz} \text { SMI } \\
\text { iView X Hi-Speed } \\
\text { eye tracker }\end{array}$ & [113] \\
\hline mean: $24.6^{\prime}$ & mean: $0.25 \mathrm{~Hz}$ & $\min : 20 \mathrm{~ms}$ & $\begin{array}{l}\min : 10 \mathrm{~ms} \\
\max : 300 \mathrm{~ms} \\
\text { mean: } 20.3 \mathrm{~ms}\end{array}$ & $\begin{array}{l}\min : 8 \% \mathrm{~s} \\
\text { peak: } 38.8 \% \mathrm{~s}\end{array}$ & & monkey & $\begin{array}{l}200 \mathrm{~Hz} \text { Scleral } \\
\text { Search Coil } \\
\text { Experiment } 1\end{array}$ & [11] \\
\hline mean: $22.6^{\prime}$ & $\overline{\text { mean: }} \overline{0} \cdot \overline{76} \overline{\mathrm{Hz}}$ & $\overline{\min } \overline{2} \overline{20 \mathrm{~ms}}$ & $\begin{array}{l}\min : \overline{10 m s} \\
\max : 300 \mathrm{~ms} \\
\text { mean: } 19.7 \mathrm{~ms}\end{array}$ & $\begin{array}{l}\min : 10 \% / \mathrm{s} \\
\text { peak: } 30.3 \% \text { s }\end{array}$ & 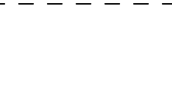 & monkey & $\begin{array}{l}200 \mathrm{~Hz} \text { Scleral } \\
\text { Search Coil } \\
\text { Experiment } 2\end{array}$ & \\
\hline $\begin{array}{l}\text { mean: } \\
21.4^{\prime}-29.0^{\prime}\end{array}$ & $\begin{array}{l}\text { mean: } \\
0.65-1.7 \mathrm{~Hz}\end{array}$ & $\overline{\min } \overline{2} \overline{20 \mathrm{~ms}}$ & $\begin{array}{l}\text { min: } 10 \mathrm{~ms} \\
\text { max: } 300 \mathrm{~ms} \\
\text { mean: } 23.9 \mathrm{~ms}\end{array}$ & $\begin{array}{l}\text { min: } 8-10 \% / \mathrm{s} \\
\text { peak: } 16.4-47.1 \% \mathrm{~s}\end{array}$ & & monkey & $\begin{array}{l}200 \mathrm{~Hz} \text { ASL6000 } \\
\text { and } 1000 \mathrm{~Hz} \\
\text { Eyelink } 1000 \\
\text { Experiment } 3\end{array}$ & \\
\hline $\begin{array}{l}\max : 2^{\circ} \\
\text { mean: } 13.7^{\prime}\end{array}$ & & & & $\min : 8^{\circ} / \mathrm{s}$ & No & monkey & $\begin{array}{l}\text { magnetic } \\
\text { induction coil }\end{array}$ & [25] \\
\hline $\max : 1.5^{\circ}$ & & & $\min : 6 \mathrm{~ms}$ & & No & human & $500 \mathrm{~Hz}$ Eyelink II & [76] \\
\hline $\begin{array}{l}\min : 3^{\prime} \\
\max : 2^{\circ}\end{array}$ & & & & $\min : 10 \% \mathrm{~s}$ & No & human & $\begin{array}{l}250 \mathrm{~Hz} \mathrm{HS} \\
\text { Video Eyetracker }\end{array}$ & [114] \\
\hline range: $1^{\prime}-36^{\prime}$ & mean: $1.08 \mathrm{~Hz}$ & min: $100 \mathrm{~ms}$ & $\min : 8 \mathrm{~ms}$ & $\max : 110 \% \mathrm{~s}$ & No & monkey & $\begin{array}{l}1000 \mathrm{~Hz} \text { eye } \\
\text { coil monocular }\end{array}$ & [61] \\
\hline $\min : 3^{\prime}-6^{\prime}$ & & & & $\min : 10 \%$ & No & monkey & $\begin{array}{l}100 \mathrm{~Hz} \mathrm{DPI} \\
\text { eye tracker } \\
\& 200 \mathrm{~Hz} \text { eye } \\
\text { coil monocular }\end{array}$ & [45] \\
\hline $\max : 1^{\circ}$ & & & $\min : 6 \mathrm{~ms}$ & & Yes & human & $\begin{array}{l}500 \mathrm{~Hz} \text { Eyelink II } \\
\text { binocular }\end{array}$ & [85] \\
\hline $\begin{array}{l}\max : 1^{\circ} \\
\operatorname{mode}: 24.9^{\prime}\end{array}$ & & & & & Yes & human & $\begin{array}{l}500 \mathrm{~Hz} \text { Eyelink II } \\
\text { binocular }\end{array}$ & [10] \\
\hline $\max : 1^{\circ}$ & & & & & & human & $\begin{array}{l}500 \mathrm{~Hz} \text { Eyelink II } \\
\text { binocular }\end{array}$ & [115] \\
\hline $\max : 1^{\circ}$ & & & $\min : 6 \mathrm{~ms}$ & & Yes & human & $\begin{array}{l}500 \mathrm{~Hz} \text { Eyelink II } \\
\text { binocular }\end{array}$ & [24] \\
\hline $\begin{array}{l}\max : 1^{\circ} \\
\text { mean: } 0.39^{\circ}\end{array}$ & mean: $0.8 \mathrm{~Hz}$ & min: $20 \mathrm{~ms}$ & mean: $12 \mathrm{~ms}$ & peak: $39 \%$ & Yes & human & $\begin{array}{l}500 \mathrm{~Hz} \text { Eyelink II } \\
\text { binocular } \\
\text { (during prolonged } \\
\text { fixation) }\end{array}$ & [14] \\
\hline $\begin{array}{l}\max : 1^{\circ} \\
\text { mean: } \\
0.55^{\circ}-0.64^{\circ}\end{array}$ & $\begin{array}{l}\text { mean: } \\
0.2-1.3 \mathrm{~Hz}\end{array}$ & min: $20 \overline{m s}$ & & & $\overline{Y e s}$ & $\overline{\text { human }} \overline{-}$ & $\begin{array}{l}500 \mathrm{~Hz} \text { Eyelink II } \\
\text { binocular } \\
\text { (during } \\
\text { free-viewing) }\end{array}$ & \\
\hline $\begin{array}{l}\max : 1^{\circ} \\
\operatorname{mean}: 0.41^{\circ}\end{array}$ & mean: $0.7 \mathrm{~Hz}$ & $\min : 20 \mathrm{~ms}$ & $\begin{array}{l}\text { min: } 6 \mathrm{~ms} \\
\text { mean: } 13 \mathrm{~ms}\end{array}$ & peak: $41 \%$ s & Yes & human & $\begin{array}{l}500 \mathrm{~Hz} \text { Eyelink II } \\
\text { binocular }\end{array}$ & [7] \\
\hline $\begin{array}{l}\max : 2^{\circ} \\
\text { mean: } 0.4^{\circ}\end{array}$ & mean: $1.0 \mathrm{~Hz}$ & min: $20 \mathrm{~ms}$ & $\begin{array}{l}\min : 6 \mathrm{~ms} \\
\text { mean: } 18 \mathrm{~ms}\end{array}$ & peak: $38^{\circ} / \mathrm{s}$ & Yes & human & $\begin{array}{l}500 \mathrm{~Hz} \text { Eyelink II } \\
\text { binocular }\end{array}$ & [8] \\
\hline \multirow[t]{2}{*}{$\begin{array}{l}\max : 1.66^{\circ} \\
\text { (observed) }\end{array}$} & & & $\min : 6 \mathrm{~ms}$ & & Yes & human & $\begin{array}{l}500 \mathrm{~Hz} \text { Eyelink II } \\
\text { binocular }\end{array}$ & [52] \\
\hline & $\begin{array}{l}\text { range: } \\
0.57-2.54 \mathrm{~Hz} \\
\text { mean: } 1.4 \mathrm{~Hz}\end{array}$ & & $\min : 12 \mathrm{~ms}$ & & No & human & $\begin{array}{l}500 \mathrm{~Hz} \text { Eyelink II } \\
\text { monocular }\end{array}$ & [84] \\
\hline \multirow[t]{3}{*}{$\max : 1.5^{\circ}$} & & & $\min : 15 \mathrm{~ms}$ & & No & human & $\begin{array}{l}200 \mathrm{~Hz} \text { Fourward } \\
\text { DPI v.6.3 } \\
\text { monocular }\end{array}$ & [82] \\
\hline & & & & & & human & 500 Hz Eyelink II & [116] \\
\hline & & & & & Yes & human & $\begin{array}{l}500 \mathrm{~Hz} \text { Eyelink II } \\
\text { binocular }\end{array}$ & [75] \\
\hline $\max : 1.5^{\circ}$ & & & $\min : 12 \mathrm{~ms}$ & $\max : 300 \% \mathrm{~s}$ & & human & $\begin{array}{l}500 \mathrm{~Hz} \text { Eyelink II } \\
\text { binocular }\end{array}$ & [77] \\
\hline \multirow[t]{2}{*}{$\max : 1.5^{\circ}$} & $\begin{array}{l}\text { mean: } \\
1.25-1.8 \mathrm{~Hz}\end{array}$ & & $\min : 12 \mathrm{~ms}$ & $\max : 300 \% \mathrm{~s}$ & Yes & human & $\begin{array}{l}500 \mathrm{~Hz} \text { Eyelink II } \\
\text { monocular and } \\
\text { binocular }\end{array}$ & [15] \\
\hline & & & & $\min : 5 \% \mathrm{~s}$ & No & monkey & $\begin{array}{l}\text { eye coil } \\
\text { monocular }\end{array}$ & [87] \\
\hline $\max : 2^{\circ}$ & & & $\min : 12 \mathrm{~ms}$ & & No & human & $\begin{array}{l}500 \mathrm{~Hz} \text { Eyelink II } \\
\text { monocular }\end{array}$ & [78] \\
\hline $\max : 1^{\circ}$ & & & $\min : 6 \mathrm{~ms}$ & & Yes & human & $\begin{array}{l}500 \mathrm{~Hz} \text { Eyelink II } \\
\text { binocular }\end{array}$ & [58] \\
\hline
\end{tabular}


Table 1 (Continued)

\begin{tabular}{|c|c|c|c|c|c|c|c|c|}
\hline Amplitude & Frequency & $\begin{array}{l}\text { Intersaccadic } \\
\text { Interval }\end{array}$ & Duration & Velocity & $\begin{array}{l}\text { Binocularity } \\
\text { required }\end{array}$ & Species & Eye Tracking & Reference \\
\hline $\begin{array}{l}\text { mean: } 0.24^{\circ} \\
\max : 2^{\circ}\end{array}$ & mean: $3.8 \mathrm{~Hz}$ & & & $\min : 3 \% \mathrm{~s}$ & No & human & $\begin{array}{l}500 \mathrm{~Hz} \text { Eyelink II } \\
\text { binocular }\end{array}$ & [6] \\
\hline \multirow[t]{3}{*}{$\max : 1^{\circ}$} & & & min: $8 \mathrm{~ms}$ & & Yes & human & $\begin{array}{l}500 \mathrm{~Hz} \text { Eyelink II } \\
\text { binocular }\end{array}$ & [42] \\
\hline & & & $\min : 12 \mathrm{~ms}$ & & Yes & human & $\begin{array}{l}250 \mathrm{~Hz} \text { Eyelink } \\
\text { binocular }\end{array}$ & [9] \\
\hline & & & & $\min : 10 \% / s$ & & & $\begin{array}{l}1000 \mathrm{~Hz} \text { infrared } \\
\text { limbus eye tracker } \\
\text { binocular } \\
\text { (horizontal only) }\end{array}$ & [117] \\
\hline $\begin{array}{l}\text { min: } 2.8^{\prime} \\
\text { mean: } 22.19^{\prime}\end{array}$ & & & min: $8 \mathrm{~ms}$ & & Yes & human & $\begin{array}{l}500 \mathrm{~Hz} \text { Eyelink II } \\
\text { binocular }\end{array}$ & [72] \\
\hline $\begin{array}{l}\max : 1.5^{\circ} \\
\max : 1^{\circ}\end{array}$ & & & & & No & human & $\begin{array}{l}500 \mathrm{~Hz} \text { Eyelink II } \\
\text { binocular }\end{array}$ & [118] \\
\hline \multirow[t]{3}{*}{$\begin{array}{l}\max : \\
15^{\prime}-18^{\prime}\end{array}$} & & & $\min : 8 \mathrm{~ms}$ & & Yes & human & $\begin{array}{l}500 \mathrm{~Hz} \text { Eyelink II } \\
\text { binocular }\end{array}$ & [83] \\
\hline & $\begin{array}{l}\text { mean: } \\
0.8-1.9 \mathrm{~Hz}\end{array}$ & & $\mathrm{~min}: 12 \mathrm{~ms}$ & & Yes & human & $\begin{array}{l}250 \mathrm{~Hz} \text { Eyelink } \\
\text { binocular }\end{array}$ & [119] \\
\hline & $\begin{array}{l}\text { mean: } \\
0.8-1.9 \mathrm{~Hz}\end{array}$ & & $\min : 8 \mathrm{~ms}$ & & Yes & human & $\begin{array}{l}500 \mathrm{~Hz} \text { Eyelink II } \\
\text { binocular }\end{array}$ & [59] \\
\hline $\max : 1^{\circ}$ & mean: $1.2 \mathrm{~Hz}$ & & $\min : 12 \mathrm{~ms}$ & & No & human & $\begin{array}{l}500 \mathrm{~Hz} \text { Eyelink II } \\
\text { monocular }\end{array}$ & [16] \\
\hline $\max : 1^{\circ}$ & & & & & & human & $500 \mathrm{~Hz}$ Eyelink II & [73] \\
\hline
\end{tabular}

Note: Unless otherwise indicated the "minimum" and "maximum" values indicate thresholds imposed by the authors to identify microsaccades. "Mean" and "range" values are the observed values in the studies.

microsaccades in the visual system, the perceptual consequences of such responses, the role of microsaccades in attention and cognition and the oculomotor mechanistic pathways that control microsaccade generation and targeting. Finally, we discuss the proposal that the visual system uses microsaccades as an optimal sampling strategy and outline the outstanding questions and directions for future research.

\section{Physical and functional properties of microsaccades}

Even though microsaccades are the largest and fastest fixational eye movement, they are relatively small in amplitude, carrying the retinal image across a range of several dozen to several hundred photoreceptor widths [4] (Box 1, and Table 1). Such small amplitudes complicate objective microsaccade characterization; thus, it is important to define the properties that microsaccades have in common with other types of eye movements, as well as those features that set them apart.

Microsaccades in monkeys are similar to those in humans [4,5,21] (see Figure I in Box 1) and have been described in other foveate vertebrates as well [22]. Importantly, microsaccades and saccades share many physical and functional characteristics, which suggests that both eye movements have a common oculomotor origin [14,23-25]. The shared properties of microsaccades and saccades can be summarized as follows (list modified from Ref. [24]):

(i) Microsaccades and saccades are generally binocular, conjugate movements with comparable amplitudes and directions in both eyes [26-28].

(ii) Microsaccades and saccades follow the main sequence. That is, microsaccade/saccade peak velocities are parametrically related to microsaccade/ saccade amplitudes [14,23] (Figure 1a), as are microsaccade/saccade durations $[7,29]$.

(iii) Visual perception thresholds are elevated during saccades and microsaccades, a phenomenon referred to as saccadic/microsaccadic suppression [11,30-35].

(iv) Intersaccadic intervals during reading are comparable to inter-microsaccadic intervals during fixation on a single letter [36].

(v) Saccade and microsaccade rates can be reduced intentionally and during specific tasks [37-40].

(vi) Voluntary saccades can be as small as fixational microsaccades [41].

(vii) Saccades and microsaccades have been linked to shifts in covert attention (see Attentional and cognitive modulation of microsaccades).

Moreover, recent research has demonstrated strong interactions in the generation of saccades and microsaccades:

(i) Microsaccade rates and amplitudes decrease before the launch of a saccade $[42,43]$.

(ii) Microsaccades occurring up to several hundred milliseconds before the saccadic start signal delay the launch of the saccade $[42,43]$.

(iii) Intersaccadic intervals are equivalent for all pairwise combinations of saccades and microsaccades during both fixation and free-viewing/visual search tasks [14].

Thus, the dichotomy between saccades and microsaccades proposed by previous studies might be fundamentally arbitrary.

The superior colliculus (SC) is a retinotopically organized structure involved in generating and controlling 


\section{Box 1. How large is a microsaccade?}

Establishment of an acceptable maximum amplitude for microsaccades poses a considerable challenge. In the 1960s and 1970s, a number of studies considered that microsaccades should have an arbitrary maximum amplitude of $\sim 12$ arcmin [112] (see Ref. [4] for a full list of microsaccade parameters in studies previous to 2004 and Ref. [14] for further discussion). However, human and primate studies in the last decade have revealed that saccades produced during fixation (i.e. microsaccades) often exceed 12 arcmin (Figure I). Thus, studies conducted in the last $5-10$ years have converged on using 1 as a practical upper threshold to characterize microsaccades (Table 1). This threshold is convenient because (even though many microsaccades are larger than 12 arcmin) the distribution of microsaccade amplitudes tends to an asymptote as it approaches $1^{\circ}$ (Figure I). A threshold of $1^{\circ}$ captures the vast majority (>90\%) of fixational saccades [8] while excluding most voluntary saccades.

It is important to note, however, that voluntary or refixation saccades can be of the same amplitude as fixational microsaccades. Thus, it is not possible to distinguish between saccades and microsaccades according to their amplitude or any other physical parameter [14]. Indeed, a recent study has proposed that both microsaccades and saccadic intrusions during fixation should be referred to as fixation saccades [80]. Recent recordings from the SC also support the existence of a microsaccade-saccade continuum, both in terms of the overlapping physical characteristics of microsaccades and saccades and their generation mechanisms [25].

The term microsaccade was coined by Zuber et al. [34]. Throughout this review we use the term microsaccade because it has become the standard phrase to designate saccades that occur during attempted fixation and thus we consider microsaccade a synonym of fixational saccade. Moreover, we propose that microsaccades should be operationally defined as saccades that are produced while attempting to fixate $[14,17]$, irrespective of their amplitude. Nevertheless, we acknowledge that an upper limit of $1^{\circ}$ is reasonable, as a rule, for practical experimental purposes (as is the case for most of the studies reviewed here).

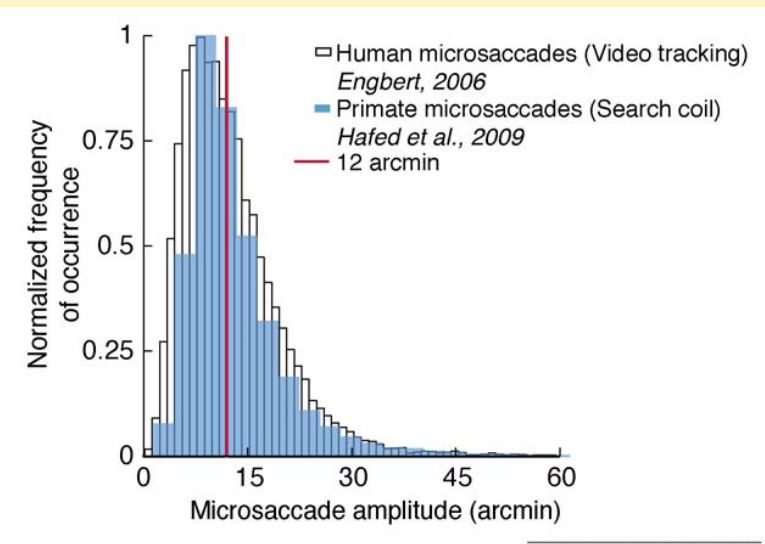

TRENDS in Neurosciences

Figure I. Distribution of microsaccade amplitudes in humans and primates. The data plots from two recent studies are superimposed [18,24]. Microsaccade amplitudes are comparable for humans and primates, irrespective of the eyetracking method used (video tracking vs. magnetic search coil). Many of the microsaccades detected are considerably larger than 12 arcmin. We should also note that prolonged visual fixation typically results in larger microsaccades than those depicted in this figure (data not shown; see Figure 3B of Ref. [14] or Figure $1 \mathrm{E}$ of Ref. [7]). Modified from Refs [18,24]. voluntary saccades. Current evidence overwhelmingly points to a key role of the SC in microsaccade production. This idea has been advanced in behavioral and computational studies $[14,24,42,43]$ but direct confirmation was only recently obtained in inactivation and electrophysiological studies in primate SC [25] (see Brain mechanisms of microsaccade generation).

\section{Visual responses to microsaccades}

Microsaccades play a critical role in maintaining visibility during fixation; thus, the neural activity evoked by microsaccades throughout the visual system can help in identifying the neural codes of visibility. Visual responses to microsaccades are the neural responses to changes in visual inputs evoked by microsaccadic displacements of the retina. Single neuron responses to microsaccades have been measured in the lateral geniculate nucleus (LGN), area V1 and several areas of the extrastriate cortex of awake monkeys. Despite some discrepancies across studies, presumably caused by a combination of differences in recording systems, characterization algorithms, visual stimuli and behavioral tasks used, there is general agreement that microsaccades primarily modulate neural activity in early visual areas through retinal motion. That is, microsaccades primarily generate neural responses by displacing the receptive fields (RFs) of visual neurons over otherwise stationary stimuli $[5,17,44,45]$ or even moving stimuli [46], as discussed below and reviewed elsewhere [4,17]. Donner and Hemilä [47] modeled the effects of microsaccades on the responses of primate retinal neurons using physiologically realistic parameters. The results suggested that microsaccades might significantly enhance sensitivity to edges, re-sharpen the image and improve spatial resolution. The results of this study support the prediction that microsaccades first generate neural activity in retinal neurons, perhaps as early as the photoreceptor level. This retinal activity might then be transmitted to the next several steps in the visual hierarchy $[4,5,44]$.

Microsaccades could help to disambiguate latency and contrast in visual perception $[4,5]$. Changes in contrast can be encoded as changes in the latency of neuronal responses $[48,49]$. The question arises as to how latency information can be used as a code for contrast without the brain first knowing the timing of events. Because the brain "knows" when a microsaccade is generated, differential latencies in visual responses might be used by the visual system to indicate differences in contrast and salience.

Microsaccades might also enhance spatial summation by synchronizing the activity of neurons with neighboring RFs. By generating bursts of spikes in visual neurons, microsaccades might moreover enhance temporal summation of responses in neurons with neighboring RFs [4].

To date, most studies addressing the effects of microsaccades on visual physiology have focused on the responses of individual neurons. However, recent research has begun to tackle the physiological effects of microsaccades on neuronal populations. Synchronous neural oscillation has been described as a signature of perceptual processes associated with object representation, attention, memory and consciousness. A recent study has found microsaccades to modulate both neuronal activity and visually induced gamma-band $(30-100 \mathrm{~Hz})$ synchronization (GBS) in primate areas V1 and V4. Microsaccade-induced 


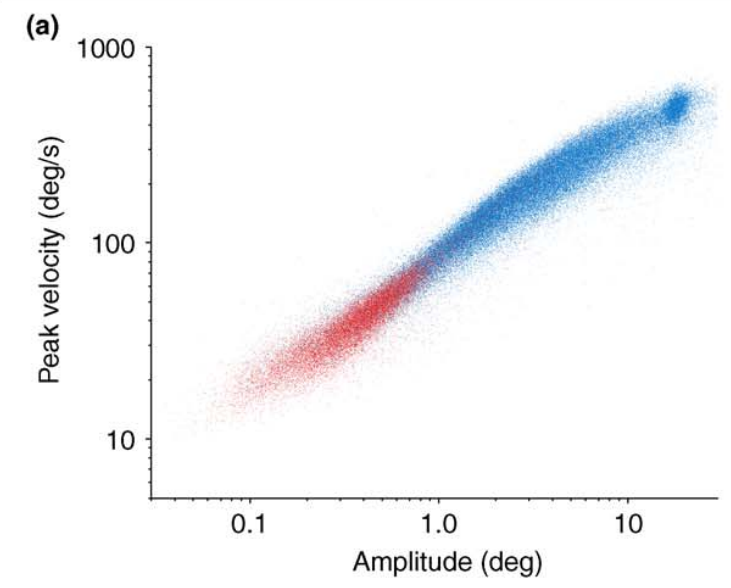

(b)

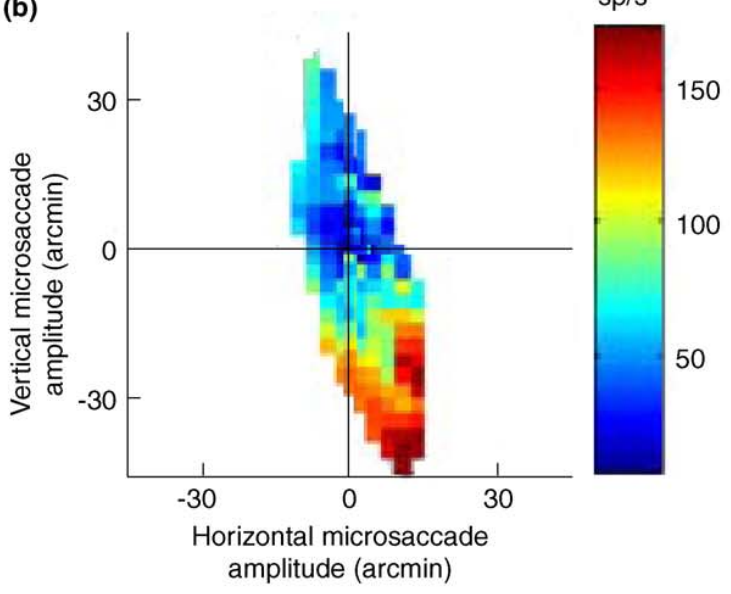

(c)

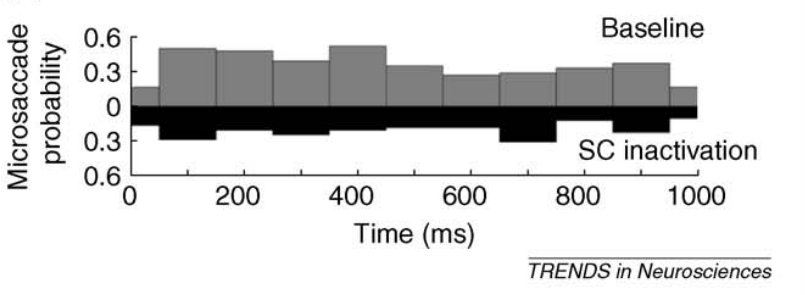

Figure 1. Microsaccade generation mechanisms. (a) Microsaccades follow the saccadic main sequence, suggesting a common generator for microsaccades and saccades. Saccades and microsaccades recorded during free-viewing (blue) follow the same main sequence as those produced during fixation (red). (Note that some blue dots are obscured by the superimposed red dots; $n=159,874$ saccades and microsaccades combined for 8 subjects. Taken from Ref. [14].) (b) Peak discharge of an SC neuron during microsaccades as a function of their horizontal and vertical amplitudes. The neuron preferred microsaccades directed to the lower right quadrant. Modified from Ref. [25]. (c) Microsaccade probability during 1s of fixation before (gray) and after (black) SC inactivation. Inactivation reduced microsaccade production. Modified from Ref. [25].

perturbations in GBS were moreover correlated with variability in behavioral response speed [50]. These results are consistent with those of an earlier study showing synchronization of neural activity due to fixational eye movements in the turtle retina [51]. Future research should further explore the modulation of synchronous activity by microsaccades along the primate visual pathway.

Yuval-Greenberg et al. [52,53] have also recently pointed out that some commonly accepted measures of
GBS might be contaminated by microsaccades in an unexpected fashion (see Ref. [54] for a counterpoint and the authors' reply in Ref. [55]). Yuval-Greenberg et al. found that the scalp-measured induced gamma-band EEG response (iGBR) largely results from small muscle artifacts associated with microsaccade production. Thus, whereas gamma-band activity might indeed play a role in brain processing (and might be modulated by microsaccades, as discussed above), care must be taken in the design and interpretation of future iGBR studies.

The study of microsaccade-driven neural activity might be pushed further in the near future thanks to recent improvements in eye-tracking technology during functional imaging. The explosion of microsaccade studies over the last decade has had much to do with the recent availability of fast and reliable eye-tracking systems for noninvasive microsaccade measurements in human subjects [4]. However, progress has been hampered by the technical limitations of obtaining non-invasive measures of brain activity during microsaccade recordings in humans. Recent efforts have succeeded in measuring BOLD signal correlates of microsaccades in human visual cortex via highspeed (1000-Hz sampling) infrared eye-tracking during fMRI. This line of enquiry should be encouraged because it will provide a much needed measure of the neural activity triggered by microsaccades throughout the human visual system, ideally in correlation with perceptual measures. Furthermore, it raises the possibility that many past fMRI results might have been arisen due to uncontrolled microsaccades, thus forcing a re-evaluation of past functional imaging data [56]. Indeed, a large amount of physiological and psychophysical visual research to date has been carried out when human and primate subjects were engaged in tasks involving visual fixation. Thus, understanding the precise physiological and perceptual contributions of microsaccades will be critical to the interpretation of previous and future research in visual neuroscience.

\section{Perceptual consequences of microsaccades}

For many decades there has been debate on whether microsaccades might preserve vision by preventing visual fading. However, no studies had directly correlated microsaccades (or any other specific fixational eye movement) to visibility. Thus, by 1980 an impasse was reached in the study of fixational eye movements $[19,57]$. Over two decades later, a direct link between microsaccade production and visual perception was finally demonstrated. To establish the correlation between microsaccades and visibility, Martinez-Conde et al. conducted a behavioral experiment in which human subjects fixated a small spot and simultaneously reported the visibility of a visual target via button press [6]. The authors found that increased microsaccade production during fixation resulted in enhanced visibility for peripheral $\left(9^{\circ}\right.$ and $6^{\circ}$ of eccentricity) and parafoveal ( $3^{\circ}$ of eccentricity) visual targets. Conversely, decreased microsaccade production led to periods of visual fading. Head restraint (or lack thereof) did not significantly change microsaccade dynamics (thus indicating that microsaccades are a natural oculomotor behavior rather than a laboratory artifact) or alter the link between micro- 
saccade production and perceptual transitions [6]. These results established a potential causal relationship between microsaccades and target visibility during fixation and corroborated predictions from physiological studies in which microsaccades increased spiking rates in V1 and LGN neurons [4]. Moreover, Engbert and Mergenthaler [58] found that microsaccades are triggered when retinal image slip is low, supporting the idea that microsaccades play a role in counteracting neural adaptation and visual fading. It should be noted that counteraction of adaptation by microsaccades does not exclude their potential involvement in fixation correction. Engbert and Kliegl [59] suggested that microsaccades counteract receptor adaptation on a short time scale and correct fixation errors on a longer time scale. Microsaccade production was subsequently linked to target visibility in other visual fading paradigms, such as the filling-in of artificial scotomas [7] (Figure 2) and motion-induced blindness [60], and to perceptual transitions in other visual phenomena, such as binocular rivalry [9] and illusory motion (perceived speed and subjective direction $[8,10])$.

Cui et al. [61] recently showed that target visibility state modulates the rate and direction of microsaccades during general flash suppression (GFS), a paradigm in which a salient monocular target disappears following the sudden presentation of a binocular surrounding pattern [62]. Cui et al. concluded that microsaccade production was affected by the percept but that the target visibility/disappearance was not caused by changes in microsaccade rates. It should be noted that the findings of Cui et al. do not contradict those of the above studies linking microsaccade production to target visibility during visual fading [6], filling-in [7], motion-induced blindness [60] or to perceptual alternations in other visual phenomena [8-10]. In all the above studies the percept changed (i.e. it undulated between two different states across time) even though the stimulus on the screen remained constant. In contrast, the study by Cui et al. involved a physical change in the stimulus (onset of the surround), which triggered disappearance of the target. Converging lines of research have shown that stimulus visibility is strongly related to transient bursts of neural activity (see Microsaccades as an optimal sampling strategy for more details). These neural transients might be triggered by sudden changes in the visual stimulus (as in the study by Cui et al.) or by sudden eye movements such as microsaccades in the case of an unchanging stimulus. Thus, one should not expect fluctuations in microsaccade production to have the same effect on GFS [61] as on perceptual alternations taking place for unchanging stimuli $[6-10,60]$.

Perceptual suppression during large saccades is known to exist [63-67] but the existence of microsaccadic suppression has been more controversial. Some studies have reported elevation of visual thresholds $[30,68]$ but others have found little or no threshold elevation during microsaccades [69,70]. Recent results by Herrington et al. [11] might represent a neural correlate of microsaccadic suppression. The authors recorded microsaccades and neural responses in middle temporal, lateral intraparietal and ventral intraparietal areas while monkeys performed motion detection tasks. Microsaccades randomly occurring

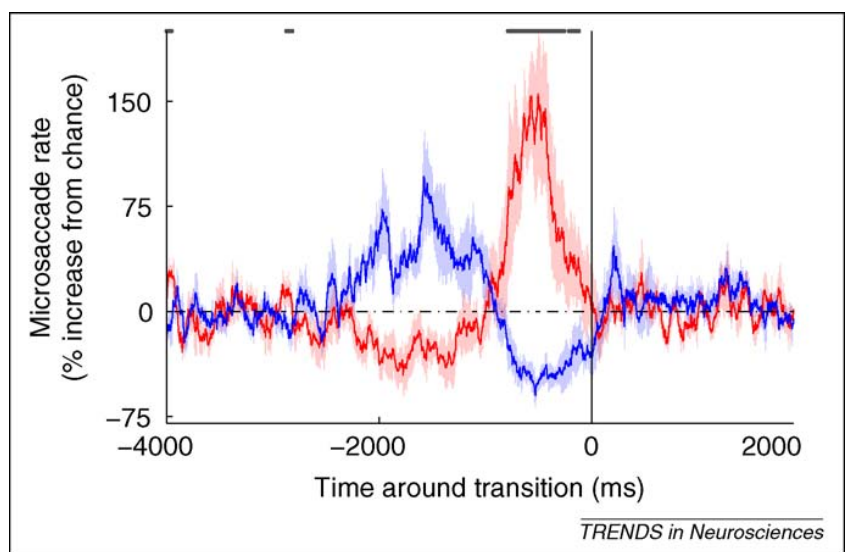

Figure 2. Microsaccades counteract visual fading and filling-in. Microsaccade rates before transitions towards visibility (unfilled scotomas, red) versus fading (filled-in scotomas, blue). The horizontal line indicates the average rate of microsaccades during the session. Shaded areas indicate SEM between subjects $(n=6)$. The gray dots at the top indicate the bins where the unfilled averages are significantly different from the filled-in averages (one-sample one-tailed paired $t$-test, $\leq 0.05$ ). From Ref. [7]. The model of Hafed et al. for microsaccade generation might explain the correlations between increases or decreases in microsaccade rates and visible or fading percepts [25].

near the time of test-stimulus onset decreased detection performance and suppressed neural activity, contributing to the correlation between neural activity and detection behavior for all three brain areas investigated (microsaccades accounted for approx. a fifth of the correlation). These findings could have important implications for future research to determine the neuronal populations underlying perceptual decisions in behaving primates (who make microsaccades during the experiments).

Future research should determine how increases and decreases in visibility triggered by microsaccades relate to different points in the lifetime of a microsaccade. For instance, decreases in visibility might occur during a microsaccade's flight, whereas enhanced visibility due to microsaccades could be driven by microsaccade termination (i.e. microsaccade "landing").

Attentional and cognitive modulation of microsaccades Recent reports have shown a link between microsaccades and cognitive processes such as attention. This association is not altogether surprising given the considerable overlap between the neural systems contributing to control of attention and control of eye movements [71]. Several studies have thus addressed the effects of shifts in spatial attention on microsaccade production. Hafed and Clark proposed that microsaccades occur because of subliminal activation of the oculomotor system by covert attention [13]. Since then, there has been consensus that microsaccade rates are modulated by both endogenous (top-down) and exogenous (bottom-up) attentional shifts, with a transient decrease in the rate of microsaccade production approximately 100-200 ms after cue onset, usually followed by a temporary enhancement $\sim 300-400 \mathrm{~ms}$ after cue onset $[8,12,13,61,72-77]$. Recent research has linked microsaccade production to other cognitive processes, such as working memory [15,76], and suggested that the absolute frequency of microsaccades is also sensitive to top-down attentional and cognitive modulations [14,78]. 
Many of these studies have predicted that the neural circuitry controlling microsaccades includes the SC, given the involvement of the SC in the targeting of large saccades, which presumably occurs in connection to shifts in attentional focus.

The modulation of microsaccade production by attention and cognition might be (at least in part) related to a role of microsaccades in enhancing visibility and preventing fading during cognitive tasks. That is, cognitive processes such as attention could modulate microsaccade generation to dynamically enhance or suppress low-level visual information at various points in time. This possibility is unexplored so far.

A number of recent papers have also found that microsaccade directions are biased towards and/or away from the spatial location suggested by an attentional cue (approx. $200 \mathrm{~ms}$ following the cue) $[12,13,18,73,75,76,79,80]$ (but see Refs $[74,81,82]$ for a counterpoint). Some of the apparent disagreement across studies might be explained by engagement of endogenous versus exogenous attention in different experimental tasks. Central informative cues (i.e. cues presented at fixation) that engage endogenous attention might produce microsaccade biases towards the peripheral location suggested by the cue $[12,13,80]$. By contrast, salient peripheral cues (visual or auditory, informative or uninformative) that engage exogenous attention might result in microsaccade biases opposite to the cue $[72,80,83]$. The observation of microsaccade biases opposite to salient and abrupt peripheral events is consistent with inhibition of return $[16,75,84,85]$.

A related topic of ongoing debate is whether biases in microsaccade directions indicate shifts in covert attention $[12,13,79]$ and/or motor programming $[42,78,82,84]$.

\section{Brain mechanisms of microsaccade generation}

Experimental evidence on the neurobiological origins of microsaccades has been sparse until very recently, constituting a major gap in our physiological understanding of these eye movements. Whereas the relationship between circuits controlling saccadic accuracy and fixation targeting is well documented [86-90], few neurophysiological studies have investigated the specific pathways responsible for the generation of microsaccades during fixation. Except for a handful of studies, most of the literature on oculomotor mechanisms of microsaccade generation is only $2-3$ years old. The studies by Van Gisbergen and colleagues in the late 1970s and early 1980s are worth noting. These authors found that putative motoneurons in the primate abducens nucleus and burst neurons in the nearby pontomedullary reticular formation (downstream of the SC) were active during saccades and microsaccades [91,92]. For decades, this was the main neurophysiological evidence of a common oculomotor mechanism underlying the generation of saccades and microsaccades.

In recent years, mounting behavioral evidence has identified the SC as a key structure in the generation of both saccades and microsaccades (see Physical and functional properties of microsaccades for further details). Recent physiological data support these predictions. Hafed and colleagues [25] made recordings from the rostral pole of the SC (which represents foveal goal locations) and found that individual neurons had particular preferences for a range of microsaccade amplitudes and locations (Figure 1b). Furthermore, the data indicated a continuous representation of saccade amplitudes and directions throughout the SC, down to the smallest microsaccades. Neuronal activity during microsaccades sometimes extended to small voluntary saccades, consistent with previous studies suggesting a microsaccade-saccade continuum $[14,23]$. Neurons active during both microsaccades and voluntary saccades usually preferred voluntary saccades smaller than $5^{\circ}$. Conversely, neurons that were active during large voluntary saccades $\left(\sim 10^{\circ}\right.$ in amplitude; more caudal in the SC map) were not active during microsaccades. Importantly, inactivation of the rostral SC led to reduced microsaccade rates, further supporting a causal role of the rostral SC in microsaccade generation [25] (Figure 1c). These results, together with the earlier behavioral studies $[14,42,43]$ and the observations that premotor neurons in the brainstem reticular formation are active during microsaccades [91,92], demonstrate that voluntary saccades and fixational microsaccades share the same neural mechanisms [14].

Hafed and colleagues also created a computer model based on the SC data to explain how shifts in covert attention may bias microsaccade directions during fixation [25]. In this model, attending to a peripheral location caused the average locus of SC activity to slightly shift towards the peripheral site, leading to a higher probability of microsaccade direction towards the attended location [25]. Hafed and colleagues concluded that microsaccade occurrence depends on the variability of SC activity representing salient goal locations and suggested that such a mechanism might also explain the link between changes in microsaccade rates and changes in visibility found by Martinez-Conde et al. [6] (Figure 2; see Perceptual consequences of microsaccades).

The recent SC experiments are an important milestone in establishing the neural mechanisms leading to microsaccade generation. Further research should aim to identify the complete oculomotor pathway and the specific circuitry involved at each neural stage.

\section{Microsaccades as an optimal sampling strategy}

Eye movements are critical to normal vision: if all eye movements are counteracted, visual perception rapidly fades due to adaptation. Thus, human eyes, as well as the eyes of other foveate vertebrates [22], are in continuous motion, with saccades and microsaccades abruptly shifting the retinal image at intervals ranging from once every several seconds to several times per second [93]. Saccades and microsaccades have comparable spatiotemporal characteristics across many varied visual tasks and viewing conditions [14]. Here we discuss how the spatiotemporal dynamics of saccades and microsaccades might reflect an optimal sampling strategy by which the brain discretely acquires visual information.

Gilchrist et al. observed that a patient who was unable to make eye movements (except for small-amplitude drifts) produced head saccades that were comparable in many of their characteristics to eye saccades in normal observers. Such similar characteristics included saccadic amplitude, 


\section{Box 2. Flashed before your eyes: transient responses to flashes versus microsaccades}

Two recent studies have shown that the responses of area $\mathrm{V} 1$ neurons to flashes are greater than, but of the same order of magnitude as, responses to microsaccades. This box reproduces the relevant plots from both studies with a matching color scheme to facilitate direct comparison (the original graphs are otherwise unchanged).

The left panel of Figure II is from Ref. [44]. The red and black curves plot the same exact V1 data, realigned to two different trigger events: flash onsets (red) and microsaccade onsets (black). See Microsaccades as an optimal sampling strategy for further details.

The right panel is from Ref. [45]. This study compared the responses to microsaccades in the presence of a stationary bar to the responses to a flashing bar shown at a different time. The paper concluded "The similarity between the responses to flashes and the activation by fixational saccades [found by Kagan et al.] contrasts sharply with a prior report indicating that flashes are about 7 times as effective as fixational saccades for V1 neurons" [45]. Here we illustrate how this apparent disagreement is reconciled by applying the same calculations to the data plots from both studies. Kagan et al. computed the ratio between the peaks for microsaccade and flash responses and concluded that the peak probability of response to microsaccades was $45 \%$ of the probability of response to flashes. This same analysis applied to the data set of Martinez-Conde et al. results in a value of $\sim 42 \%$. Martinez-Conde et al. considered the differing baselines of microsaccade and flash response plots; thus, they compared the peakto-baseline relation for each curve. This produced a value of 7.6 (meaning that the peak increase in spike probability after a flash was about seven-fold higher than the peak increase in spike probability after a microsaccade). This analysis applied to the Kagan et al. study results in a value of $\sim 7.1$. Thus, the results from both studies are equivalent and indeed support each other. Alternative quantifications are also possible. Perhaps a better metric, which was not calculated by either study, would be to subtract the baseline from each peak response and then calculate the ratios for the resultant values. More importantly, the results from both studies are consistent with each other, regardless of the specific metrics used to compare them and despite their methodological differences.
Martinez-Conde et (2002)

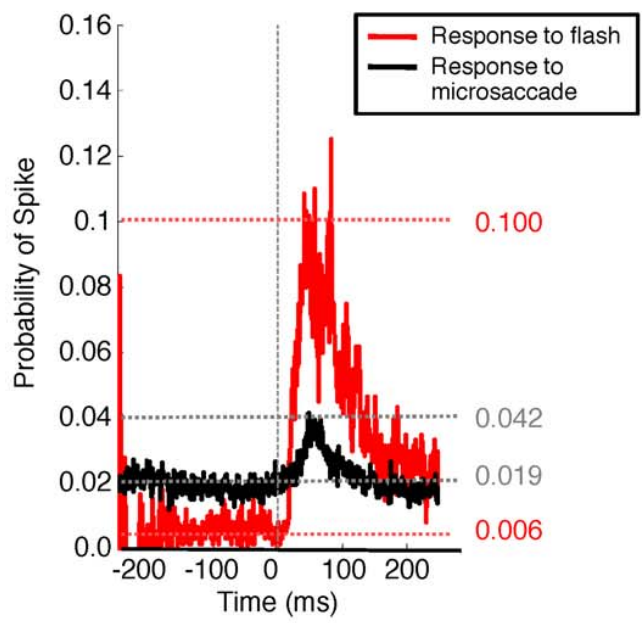

Kagan et al (2008)

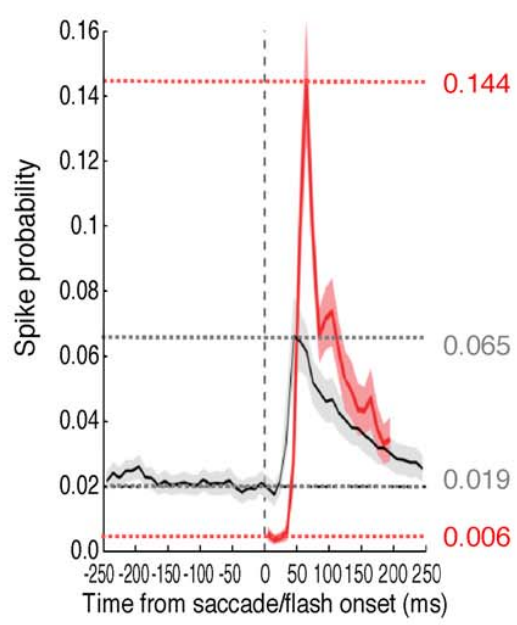

\begin{tabular}{l}
\cline { 2 - 3 } \multicolumn{1}{c|}{ Kagan et al metric } \\
\cline { 2 - 3 } \multicolumn{1}{c|}{$\begin{array}{c}\text { Martinez-Conde et al } \\
\text { data }\end{array}$}
\end{tabular}

\begin{tabular}{l}
\cline { 2 - 3 } \multicolumn{1}{c|}{ Martinez-Conde et al metric } \\
\cline { 2 - 3 } \multicolumn{1}{c|}{$\begin{array}{c}\text { Martinez-Conde et al } \\
\text { data }\end{array}$}
\end{tabular}

TRENDS in Neurosciences

Figure II. Neural responses to flashes versus microsaccades in Martinez-Conde et al. 2002 [44] (left panel) and Kagan et al. 2008 [45] (right panel). The accompanying tables confirm that the results from both studies are consistent with each other, regardless of the specific metrics used to compare them. The calculations were directly estimated from the population graphs in both papers (the results published by Martinez-Conde et al. [44] were first calculated numerically for each neuron, and then averaged across neurons).

the duration of the intersaccadic intervals, the length of intervening fixations and the range of visual scanning during exploration of pictures, although the peak velocity of head saccades was slower than that of regular eye saccades. These head saccades enabled the patient to read at normal speed and even perform complicated visuomotor tasks, such as making a cup of tea, with no problems. Although microsaccades were not tested per se, the authors concluded that "saccadic movements, of the head or the eye, form the optimal sampling method for the brain" 
(compared, for example, to smooth scanning of the visual scene) [94-96].

The idea that saccades and microsaccades discretely sample visual information is further supported by physiological studies comparing neuronal responses triggered by saccades/microsaccades to responses triggered by instantaneous events, such as blinks and flashes (Box 2). Gawne and Martin measured the responses of neurons in V1, V2, $\mathrm{V} 3 \mathrm{~V} / \mathrm{VP}$ and $\mathrm{V} 4 \mathrm{~V}$ to the onset and termination of visual stimuli elicited by flashes, blinks and saccades. Although a minority of neurons presented responses that varied as a function of the neural event, most of them showed similar responses regardless of condition, suggesting that the neural circuitry underlying visual perception responds to different transient events in a similar manner [97].

Martinez-Conde et al. [44] compared the transient responses to microsaccade onset to those generated by visual flash onset in 54 neurons of the LGN $(n=48)$ and V1 $(n=6)$. A periodic flashing bar (on for $1 \mathrm{~s}$, off for $1 \mathrm{~s}$ ) was presented over the RF of a neuron while the monkey fixated a cross. Responses to microsaccades that occurred while the flashing bar was on were directly compared to responses to flash onsets that occurred while the monkey produced microsaccades. The effectiveness of each microsaccade thus depended on the relative positions of the bar and the $\mathrm{RF}$ at the time of microsaccade onset. Likewise, the effectiveness of each flash depended on the relative positions of the bar and the $\mathrm{RF}$ at flash onset. Because of ongoing microsaccades and other eye movements, sometimes the flashing bar turned on exactly on top of the RF and sometimes it was displaced with respect to the $R F$ center. Thus, any spatial shifts between the bar and the RF due to eye movements were equivalent across the microsaccade and flash response conditions. This experimental design facilitated direct statistical comparison of microsaccade- and flash-triggered responses in the same neurons and at the same time. Neural responses to flashes were stronger than but of the same order of magnitude as responses to microsaccades, perhaps because of the relative abruptness of flashes with respect to microsaccades (see Figure II in Box 2, left panel). Firing modulations caused by microsaccades could thus be equated to those caused by the reappearance or reflashing of the visual stimulus, albeit with a weaker neuronal response.

Transient responses evoked by microsaccades in primate visual neurons often take the form of bursts of spikes $[4,5,44]$. These might or might not be accompanied by sustained firing during intersaccadic periods [45]. Bursty firing effectively indicates the presence of previous microsaccades in the awake fixating primate, suggesting that this type of neural activity might be highly conducive to sustaining a visible image during fixation $[5,17,44]$. In agreement with this idea, recent research has shown that microsaccades counteract perceptual fading during fixation [6,7] and might lead to more efficient sampling of spatial detail [47]. Furthermore, the suppression of transient bursts of activity has been related to perceptual suppression during blinks [98] and to decreased target visibility in visual masking paradigms [99-101]. Other studies suggest that V1 neurons produce stronger responses to transient than to drifting stimuli. Such neural transients might underlie the behavior of cortical neurons as coincidence detectors [102,103].

Perceptual experiments have also shown that slow gradual changes (that presumably result in sustained neural firing) are difficult to detect, even in the absence of interruptions or distractions [104]. These results further support the notion that discrete temporal sampling might be an optimal strategy for visual perception.

Discrete temporal sampling might be optimal across a number of sensory systems. Sniffs in rodent olfaction discretely sample sensory information every $200-300 \mathrm{~ms}$ and thus are similar in their temporal dynamics to primate saccades [14,105] and microsaccades [14]. A similar mode of discrete sampling might also be present when objects are recognized through tactile information, for instance when subjects use their fingertips to identify an object with their eyes closed or when blind individuals read Braille script. Uchida et al. [105] suggested that discrete sensory sampling might be evolutionarily advantageous because it could speed up information processing (i.e. limiting the processing of low-level information to short chunks could facilitate rapid construction of global perceptual images). Thorpe et al. showed that the visual processing required to determine the gist of a briefly flashed natural scene can be achieved in $150 \mathrm{~ms}$ [106]. This interval could not be shortened, even after extensive training; thus, there might be a limit to the number of neural stages and speed involved in the processing of visual information [107]. The generation of a saccade every $200-300$ s could provide multiple individual high-acuity snapshots of a visual scene [105] (intersaccadic intervals are equivalent for saccades and microsaccades [14]). However, because of the limitations in visual processing speed stated above, faster rates of saccade/microsaccade production might not significantly improve vision.

Finally, microsaccades might not be randomly produced in time, but triggered dynamically as a function of low retinal slip [58]. Future research should investigate whether microsaccades dynamically displace retinal images with a temporal structure that serves to overcome adaptation within a discrete sampling framework.

\section{Summary and conclusions}

Our aims in this review were to discuss the most recent advances in microsaccade research within a neurophysiological framework, to analyze how disparate discoveries in different areas of microsaccade research impact other findings and to identify current trends in the field. We first focused on the physical and functional properties of microsaccades and established that microsaccades and saccades must share a common oculomotor origin. We then discussed the neural responses to the retinal image displacements due to microsaccades and pointed to research showing that microsaccades evoke bursts of neural activity and strong synchronized responses in the early visual system. We reviewed recent studies that have linked perception directly to microsaccades for the first time, as well as the role of microsaccades in attention and cognition. We discussed the oculomotor evidence demonstrating the mechanistic pathways that control the common generation of microsaccades and saccades, some of which have been elucidated in recent neurophysiological studies of the SC. 
Finally, we proposed that microsaccades and saccades might represent an optimal discrete temporal sampling strategy for the visual system.

\section{Outstanding questions and directions for future research}

The study of microsaccades is one of the fastest growing fields in contemporary neuroscience. However, some important gaps remain that would benefit from directed research efforts. Here we point out several areas of interest that are not discussed elsewhere in this review and some of the most promising current research directions.

\section{Effect of microsaccades in central vision}

Microsaccades have been linked to enhanced visibility of peripheral and parafoveal stimuli [6] and they generate visual responses in RFs at all eccentricities [44]. The foveal image change resulting from a microsaccade can also determine percept dominance in binocular rivalry [9]. However, the perceptual impact of microsaccades in foveal vision has not been directly investigated. Because foveal vision may be sustained in the absence of microsaccades, it has been argued that the perceptual role of microsaccades solely concerns the visual periphery. However, even if drifts and/or tremor can maintain foveal vision on their own, this does not rule out the possibility that microsaccades also have a role in foveal vision. Thus, if drifts and tremor were eliminated microsaccades alone might sustain foveal vision during fixation [4]. Future research should determine the perceptual consequences of the interaction between microsaccade amplitudes and receptive field sizes at varying retinal eccentricities, including those in the foveal range.

\section{Effects of microsaccades in various visual phenomena} Microsaccade dynamics are related to perceptual transitions in visual fading [6], filling-in [7], illusory motion $[8,10]$ and binocular rivalry [9]. Many other visual illusions are attenuated or even disappear when the observer fixates carefully and thus suppresses microsaccades. Therefore, microsaccades might drive (completely or partially) the generation of such illusory percepts [17]. Future research should determine the perceptual effects that are modulated by microsaccades and relate them to the underlying neural circuits.

\section{Neural correlates of microsaccadic suppression}

The world remains perceptually stable during microsaccades despite the fact that they cause sizable retinal motion that should be easily resolvable. Some studies have reported elevation of visual thresholds during microsaccades (i.e. microsaccadic suppression) but the neural correlates of this perceptual phenomenon are not well understood [4] (but see Ref. [11]). Furthermore, it is not known how perceptual suppression during microsaccades might interact temporally with the visibility enhancement also brought about by microsaccades $[6,7]$.

\section{Neural consequences of the attentional modulation of microsaccades}

In the last few years numerous studies have investigated how microsaccade rates and/or directions are modulated by attention in a variety of tasks. However, the physiological consequences of such attentional modulation remain fundamentally unexplored. Furthermore, although the neural effects of increased attention as opposed to increased microsaccade production can be separated [108], it is possible that some neural and perceptual effects currently attributed to attention are, at least partially, due to dynamic changes in microsaccade production during attentional tasks. Future experiments should trace the neural pathways by which attention, cognition and microsaccades can interact.

\section{Extraretinal modulation of neural responses by microsaccades}

Microsaccades generate neural responses in early visual neurons by displacing their receptive fields over otherwise stationary stimuli. However, such clear-cut retinogeniculate cortical responses might be accompanied by less evident extraretinal modulations. Some recent studies suggest that microsaccade-driven extraretinal modulations are present in a minority of neurons in area V1, but the evidence is conflicting as to the sign of such modulations (inhibitory, excitatory or both) and their timing with respect to the primary retinal responses generated by microsaccades [4]. Different groups have found: (a) suppression of firing associated with microsaccade onset in a marginal percentage of V1 neurons [5]; (b) suppression of firing after microsaccades in about a third of V1 neurons [109] (although this group later stated that microsaccades increase activity in area V1 [110]); and (c) weak suppression of firing after microsaccade onset followed by stronger enhancement 100-200 ms later in a third of V1 neurons [45]. In every study the apparent extraretinal responses were much smaller than the neural responses explained by straightforward retinal activation. Future research should ascertain the potential extraretinal modulations contributed by microsaccades throughout the visual pathway and determine their neural origins (i.e. corollary discharge from oculomotor centers of the brain, top-down attentional feedback, etc) and perceptual consequences (e.g. as a neural correlate of perceptual suppression during microsaccades).

\section{Microsaccades in visual and neural pathologies}

Insufficient fixational eye movements lead to neural adaptation and fading, whereas excessive eye motion produces blurring and unstable vision during fixation [17]. Thus everyday vision achieves a very delicate balance. Even when we explore or search a visual scene, we fixate our gaze (in between large exploratory saccades) for $80 \%$ of the time $[14,17]$. However, few studies have focused on pathological fixational eye movements as a sign of or contributor to visual or neural disease [17,111]. Future research should assess the possible impairment of microsaccades and other fixational eye movements in central and peripheral pathologies and the potential implications for treatment and/or early diagnosis.

\section{Acknowledgements}

We thank Jorge Otero-Millan for scientific discussion and help with the figures and Isabel Gomez-Caraballo and Manuel Ledo for technical 
assistance. This work was funded by grants from the National Science Foundation (award 0643306 to SMC), the Arizona Biomedical Research Commission (award 07-102 to SMC), the Dana Foundation Program in Brain and Immuno-Imaging to SMC, and the Barrow Neurological Foundation to SMC and SLM. XGT was a fellow of the Caja Madrid Foundation. The authors have no conflict of interest and nothing to disclose.

\section{References}

1 Riggs, L.A. and Ratliff, F. (1952) The effects of counteracting the normal movements of the eye. J. Opt. Soc. Am. 42, 872-873

2 Ditchburn, R.W. and Ginsborg, B.L. (1952) Vision with a stabilized retinal image. Nature 170, 36-37

3 Yarbus, A.L. (1967) Eye Movements and Vision. Plenum Press

4 Martinez-Conde, S. et al. (2004) The role of fixational eye movements in visual perception. Nat. Rev. Neurosci. 5, 229-240

5 Martinez-Conde, S. et al. (2000) Microsaccadic eye movements and firing of single cells in the striate cortex of macaque monkeys. Nat. Neurosci. 3, 251-258

6 Martinez-Conde, S. et al. (2006) Microsaccades counteract visual fading during fixation. Neuron 49, 297-305

7 Troncoso, X. et al. (2008) Microsaccades counteract perceptual fillingin, J. Vis. 8(14):15, 1-9

8 Troncoso, X. et al. (2008) Microsaccades drive illusory motion in the enigma illusion. Proc. Natl. Acad. Sci. U. S. A. 105, 16033-16038

9 van Dam, L.C. and van Ee, R. (2006) Retinal image shifts, but not eye movements per se, cause alternations in awareness during binocular rivalry. J. Vis. 6(11):3, 1172-1179

10 Laubrock, J. et al. (2008) Fixational eye movements predict the perceived direction of ambiguous apparent motion. J. Vis. 8(14):13, $1-17$

11 Herrington, T.M. et al. (2009) The effect of microsaccades on the correlation between neural activity and behavior in middle temporal, ventral intraparietal, and lateral intraparietal areas. $J$. Neurosci. 29, 5793-5805

12 Engbert, R. and Kliegl, R. (2003) Microsaccades uncover the orientation of covert attention. Vis. Res. 43, 1035-1045

13 Hafed, Z.M. and Clark, J.J. (2002) Microsaccades as an overt measure of covert attention shifts. Vis. Res. 42, 2533-2545

14 Otero-Millan, J. et al. (2008) Saccades and microsaccades during visual fixation, exploration and search: foundations for a common saccadic generator. J. Vis. 8(14):21, 1-18

15 Valsecchi, M. et al. (2007) Visual oddballs induce prolonged microsaccadic inhibition. Exp. Brain Res. 177, 196-208

16 Galfano, G. et al. (2004) Inhibition of return in microsaccades. Exp. Brain Res. 159, 400-404

17 Martinez-Conde, S. (2006) Fixational eye movements in normal and pathological vision. Prog. Brain Res. 154, 151-176

18 Engbert, R. (2006) Microsaccades: a microcosm for research on oculomotor control, attention, and visual perception. Prog. Brain Res. 154, 177-192

19 Kowler, E. and Steinman, R.M. (1980) Small saccades serve no useful purpose: reply to a letter by R. W. Ditchburn. Vis. Res. 20, 273-276

20 Martinez-Conde, S. and Macknik, S.L. (2007) Windows on the mind. Scientific American. 297, 56-63

21 Steinman, R.M. et al. (1973) Miniature eye movement. Science 181, 810-819

22 Martinez-Conde, S. and Macknik, S.L. (2008) Fixational eye movements across vertebrates: comparative dynamics, physiology, and perception. J. Vis. 8(14):28, 1-16

23 Zuber, B.L. and Stark, L. (1965) Microsaccades and the velocityamplitude relationship for saccadic eye movements. Science 150, 1459-1460

24 Rolfs, M. et al. (2008) Toward a model of microsaccade generation: the case of microsaccadic inhibition. J. Vis. 8(11):5, 1-23

25 Hafed, Z.M. et al. (2009) A neural mechanism for microsaccade generation in the primate superior colliculus. Science 323, 940-943

26 Ditchburn, R.W. and Ginsborg, B.L. (1953) Involuntary eye movements during fixation. J. Physiol. 119, 1-17

27 Krauskopf, J. et al. (1960) Analysis of eye movements during monocular and binocular fixation. J. Opt. Soc. Am. 50, 572-578

28 Lord, M.P. (1951) Measurement of binocular eye movements of subjects in the sitting position. Brit. J. Ophthalmol. 35, 21-30
29 Bahill, A.T. et al. (1975) The main sequence, a tool for studying human eye movements. Math. Biosci. 24, 191-204

30 Beeler, G.W. (1967) Visual threshold changes resulting from spontaneous saccadic eye movements. Vis. Res. 7, 769-775

31 Latour, P.L. (1962) Visual threshold during eye movements. Vis. Res. $2,261-262$

32 Volkmann, F.C. (1962) Vision during voluntary saccadic eye movements. J. Opt. Soc. Am. 52, 571-578

33 Volkmann, F.C. et al. (1968) Time course of visual inhibition during voluntary saccades. J. Opt. Soc. Am. 58, 562-569

34 Zuber, B.L. et al. (1964) Saccadic suppression associated with microsaccades. Q. Prog. Rep. 74, 244-249

35 Zuber, B.L. and Stark, L. (1966) Saccadic suppression: elevation of visual threshold associated with saccadic eye movements. Exp. Neurol. 16, 65-79

36 Cunitz, R.J. and Steinman, R.M. (1969) Comparison of saccadic eye movements during fixation and reading. Vis. Res. 9, 683-693

37 Fiorentini, A. and Ercoles, A.M. (1966) Involuntary eye movements during attempted monocular fixation. Atti Fond. Giorgio Ronchi 21, 199-217

38 Steinman, R.M. et al. (1967) Voluntary control of microsaccades during maintained monocular fixation. Science 155, 1577-1579

39 Bridgeman, B. and Palca, J. (1980) The role of microsaccades in high acuity observational tasks. Vis. Res. 20, 813-817

40 Winterson, B.J. and Collewijn, H. (1976) Microsaccades during finely guided visuomotor tasks. Vis. Res. 16, 1387-1390

41 Haddad, G.M. and Steinman, R.M. (1973) The smallest voluntary saccade: implications for fixation. Vis. Res. 13, 1075-1086

42 Rolfs, M. et al. (2006) Shortening and prolongation of saccade latencies following microsaccades. Exp. Brain Res. 169, 369-376

43 Rolfs, M. et al. (2008) Microsaccade-induced prolongation of saccadic latencies depends on microsaccade amplitude. J. Eye Move. Res. 1(3):1, 1-8

44 Martinez-Conde, S. et al. (2002) The function of bursts of spikes during visual fixation in the awake primate lateral geniculate nucleus and primary visual cortex. Proc. Natl. Acad. Sci. U. S. A. 99 13920-13925

45 Kagan, I. et al. (2008) Saccades and drifts differentially modulate neuronal activity in V1: effects of retinal image motion, position, and extraretinal influences. J. Vis. 8(14):19, 11-25

46 Bair, W. and O'Keefe, L.P. (1998) The influence of fixational eye movements on the response of neurons in area MT of the macaque. Vis. Neurosci. 15, 779-786

47 Donner, K. and Hemilä, S. (2007) Modelling the effect of microsaccades on retinal responses to stationary contrast patterns. Vis. Res. 47, 1166-1177

48 Gawne, T.J. et al. (1996) Latency: another potential code for feature binding in striate cortex. J. Neurophysiol. 76, 1356-1360

49 Albrecht, D.G. and Hamilton, D.B. (1982) Striate cortex of monkey and cat: contrast response function. J. Neurophysiol. 48, 217-237

50 Bosman, C.A. et al. (2009) A microsaccadic rhythm modulates gammaband synchronization and behavior. J. Neurosci. 29, 9471-9480

51 Greschner, M. et al. (2002) Retinal ganglion cell synchronization by fixational eye movements improves feature stimation. Nat. Neurosci. 5, 341-347

52 Yuval-Greenberg, S. et al. (2008) Transient induced gamma-band response in EEG as a manifestation of miniature saccades. Neuron $58,429-441$

53 Yuval-Greenberg, S. and Deouell, L.Y. (2009) The broadbandtransient induced gamma-band response in scalp EEG reflects the execution of saccades. Brain Topogr. 22, 3-6

54 Melloni, L. et al. (2009) Response to: Yuval-Greenberg et al., "Transient induced gamma-band response in EEG as a manifestation of miniature saccades." Neuron 58, 429-441. Neuron 62, 8-10

55 Yuval-Greenberg, S. et al. (2009) Response to letter: Melloni et al., "Transient induced gamma-band response in EEG as a manifestation of miniature saccades." Neuron 58, 429-441. Neuron $62,10-12$

56 Tse, P.U. et al. (2007) fMRI BOLD signal reveals neural correlates of microsaccades. J. Vis. 7 (9), 318a

57 Ditchburn, R.W. (1980) The function of small saccades. Vis. Res. 20, 271-272 
58 Engbert, R. and Mergenthaler, K. (2006) Microsaccades are triggered by low retinal image slip. Proc. Natl. Acad. Sci. U. S. A. 103, 71927197

59 Engbert, R. and Kliegl, R. (2004) Microsaccades keep the eyes' balance during fixation. Psychol. Sci. 15, 431-436

60 Hsieh, P.J. and Tse, P.U. (2009) Microsaccade rate varies with subjective visibility during motion-induced blindness. PLoS ONE 4, e5163

61 Cui, J. et al. (2009) Visibility states modulate microsaccade rate and direction. Vis. Res. 49, 228-236

62 Wilke, M. et al. (2003) Generalized flash suppression of salient visual targets. Neuron 39, 1043-1052

63 Bridgeman, B.B. and Macknik, S.L. (1995) Saccadic suppression relies on luminance information. Psychol. Res. 58, 163-168

64 Macknik, S.L. et al. (1991) Flicker distorts visual space constancy. Vis. Res. 31, 2057-2064

65 Wurtz, R.H. (1968) Visual cortex neurons: response to stimuli during rapid eye movements. Science 162, 1148-1150

66 Wurtz, R.H. (1969) Comparison of effects of eye movements and stimulus movements on striate cortex neurons of the monkey. $J$. Neurophysiol. 32, 987-994

67 Ross, J. et al. (2001) Changes in visual perception at the time of saccades. Trends Neurosci. 24, 113-121

68 Ditchburn, R.W. (1955) Eye-movements in relation to retinal action. Opt. Acta (Lond.) 1, 171-176

69 Krauskopf, J. (1966) Lack of inhibition during involuntary saccades. Am. J. Psychol. 79, 73-81

70 Sperling, G. (1990) Comparison of perception in the moving and stationary eye. In Eye Movements and Their Role in Visual and Cognitive Processes (Kowler, E., ed.), pp. 307-351, Elsevier

71 Corbetta, M. et al. (1998) A common network of functional areas for attention and eye movements. Neuron 21, 761-773

72 Laubrock, J. et al. (2005) Microsaccade dynamics during covert attention. Vis. Res. 45, 721-730

73 Rolfs, M. et al. (2004) Microsaccade orientation supports attentional enhancement opposite a peripheral cue: commentary on Tse, Sheinberg, and Logothetis (2003). Psychol. Sci. 15, 431-436

74 Tse, P.U. et al. (2004) The distribution of microsaccade directions need not reveal the location of attention. Psychol. Sci. 15, 708710

75 Turatto, M. et al. (2007) Microsaccades distinguish between global and local visual processing. Neuroreport 18, 1015-1018

76 Valsecchi, M. and Turatto, M. (2009) Microsaccadic responses in a bimodal oddball task. Psychol. Res. 73, 23-33

77 Valsecchi, M. and Turatto, M. (2007) Microsaccadic response to visual events that are invisible to the superior colliculus. Behav. Neurosci. 121, 786-793

78 Betta, E. and Turatto, M. (2006) Are you ready? I can tell by looking at your microsaccades. Neuroreport 17, 1001-1004

79 Laubrock, J. et al. (2007) Microsaccades are an index of covert attention: commentary on Horowitz, Fine, Fencsik, Yurgenson, and Wolfe (2007). Psychol. Sci. 18, 364-366

80 Gowen, E. et al. (2007) Modulation of saccadic intrusions by exogenous and endogenous attention. Brain Res. 1141, 154-167

81 Horowitz, T.S. et al. (2007) Microsaccades and attention: does a weak correlation make an index? Reply to Laubrock, Engbert, Rolfs, and Kliegl (2007). Psychol. Sci. 18, 367-368

82 Horowitz, T.S. et al. (2007) Fixational eye movements are not an index of covert attention. Psychol. Sci. 18, 356-363

83 Rolfs, M. et al. (2005) Crossmodal coupling of oculomotor control and spatial attention in vision and audition. Exp. Brain Res. 166, 427-439

84 Betta, E. et al. (2007) Microsaccadic response during inhibition of return in a target-target paradigm. Vis. Res. 47, 428-436

85 Kliegl, R. et al. (2009) Microsaccadic modulation of response times in spatial attention tasks. Psychol. Res. 73, 136-146

86 Hafed, Z.M. et al. (2008) Superior colliculus inactivation causes stable offsets in eye position during tracking. J. Neurosci. 28, 8124-8137

87 Goffart, L. et al. (2006) Influence of background illumination on fixation and visually guided saccades in the rhesus monkey. Vis. Res. 46, 149-162
88 Goffart, L. et al. (2004) Deficits in saccades and fixation during muscimol inactivation of the caudal fastigial nucleus in the rhesus monkey. J. Neurophysiol. 92, 3351-3367

89 Quinet, J. and Goffart, L. (2007) Head-unrestrained gaze shifts after muscimol injection in the caudal fastigial nucleus of the monkey. $J$. Neurophysiol. 98, 3269-3283

90 Munoz, D.P. and Wurtz, R.H. (1993) Fixation cells in monkey superior colliculus. I. Characteristics of cell discharge. J. Neurophysiol. 70, 559-575

91 Van Gisbergen, J.A. et al. (1981) A quantitative analysis of generation of saccadic eye movements by burst neurons. J. Neurophysiol. 45, 417442

92 Van Gisbergen, J.A.M. and Robinson, D.A. (1977) Generation of micro and macrosaccades by burst neurons in the monkey. In Control of Gaze by Brain Stem Neurons (Baker, R. and Berthoz, A., eds), pp. 301-308, Elsevier/North-Holland

93 Martinez-Conde, S. et al. (2008) Eye movements and the perception of a clear and stable visual world. J. Vis. 8(14):i, 1

94 Gilchrist, I.D. et al. (1997) Saccades without eye movements. Nature $390,130-131$

95 Gilchrist, I.D. et al. (1998) Using the eye-movement system to control the head. Proc. R. Soc. Lond. B Biol. Sci. 265, 1831-1836

96 Land, M.F. et al. (2002) The organization of visually mediated actions in a subject without eye movements. Neurocase $8,80-87$

97 Gawne, T.J. and Martin, J.M. (2002) Responses of primate visual cortical neurons to stimuli presented by flash, saccade, blink, and external darkening. J. Neurophysiol. 88, 2178-2186

98 Gawne, T.J. and Martin, J.M. (2000) Activity of primate V1 cortical neurons during blinks. J. Neurophysiol. 84, 2691-2694

99 Macknik, S.L. et al. (2000) The role of spatiotemporal edges in visibility and visual masking. Proc. Natl. Acad. Sci. U. S. A. 97 7556-7560

100 Macknik, S.L. and Livingstone, M.S. (1998) Neuronal correlates of visibility and invisibility in the primate visual system. Nat. Neurosci. 1, 144-149

101 Macknik, S.L. and Martinez-Conde, S. (2004) The spatial and temporal effects of lateral inhibitory networks and their relevance to the visibility of spatiotemporal edges. Neurocomputing 58-60 775782

102 Williams, P.E. and Shapley, R.M. (2007) A dynamic nonlinearity and spatial phase specificity in macaque V1 neurons. J. Neurosci. 27 5706-5718

103 Shelley, M. et al. (2002) States of high conductance in a large-scale model of the visual cortex. J. Comput. Neurosci. 13, 93-109

104 Simons, D.J. et al. (2000) Change blindness in the absence of a visual disruption. Perception 29, 1143-1154

105 Uchida, N. et al. (2006) Seeing at a glance, smelling in a whiff: rapid forms of perceptual decision making. Nat. Rev. Neurosci. 7, 485-491

106 Thorpe, S. et al. (1996) Speed of processing in the human visual system. Nature $381,520-522$

107 Fabre-Thorpe, M. et al. (2001) A limit to the speed of processing in ultra-rapid visual categorization of novel natural scenes. J. Cogn. Neurosci. 13, 171-180

108 Chen, Y. et al. (2008) Task difficulty modulates the activity of specific neuronal populations in primary visual cortex. Nat. Neurosci. 11, 974982

109 Leopold, D.A. and Logothetis, N.K. (1998) Microsaccades differentially modulate neural activity in the striate and extrastriate visual cortex. Exp. Brain Res. 123, 341-345

110 Moutoussis, K. et al. (2005) A binocular rivalry study of motion perception in the human brain. Vis. Res. 45, 2231-2243

111 Serra, A. et al. (2008) Suppression of saccadic intrusions in hereditary ataxia by memantine. Neurology $70,810-812$

112 Collewijn, H. and Kowler, E. (2008) The significance of microsaccades for vision and oculomotor control. J. Vis. 8(14):20, 1-21

113 Valsecchi, M. et al. (2009) Microsaccadic inhibition and P300 enhancement in a visual oddball task. Psychophysiology 46, 635-644

114 Beer, A.L. et al. (2008) A motion illusion reveals mechanisms of perceptual stabilization. PLoS ONE 3, e2741

115 Moshel, S. et al. (2008) Persistence and phase synchronization properties of fixational eye movements. Eur. Phys. J. Special Top. $161,207-223$ 
116 Mergenthaler, K. and Engbert, R. (2007) Modeling the control of fixational eye movements with neurophysiological delays. Phys. Rev. Lett. 98, 138104

117 Murakami, I. et al. (2006) A positive correlation between fixation instability and the strength of illusory motion in a static display. Vis. Res. 46, 2421-2431
118 Liang, J.R. et al. (2005) Scaling of horizontal and vertical fixational eye movements. Phys. Rev. E 71, 031909

119 van Dam, L.C. and van Ee, R. (2005) The role of (micro)saccades and blinks in perceptual bi-stability from slant rivalry. Vis. Res. 45, 2417-2435 